\title{
PHYSIOLOGICAL AND MORPHOLOGICAL PROPERTIES OF, AND EFFECT OF SUBSTANCE P ON, NEURONS IN THE A7 CATECHOLAMINE CELL GROUP IN RATS
}

\author{
M.-Y. MIN, ${ }^{a, b}$ Y.-W. WU, ${ }^{b}$ P.-Y. SHIH, ${ }^{b}$ H.-W. LU, ${ }^{b}$ \\ C.-C. LIN, ${ }^{b}$ Y. WU, ${ }^{b}$ M.-J. LI ${ }^{\mathrm{a}}$ AND H.-W. YANG ${ }^{\mathrm{c} *}$ \\ ${ }^{a}$ Department of Life Science, College of Life Science, National Taiwan \\ University, No. 1, Sec. 4 Roosevelt Road, Taipei 106, Taiwan \\ ${ }^{b}$ Institute of Zoology, College of Life Science, National Taiwan Univer- \\ sity, Taipei 106, Taiwan \\ ${ }^{c}$ Department of Biomedical Sciences, Chung-Shan Medical University, \\ No. 110, Sec. 1 Chien-Kuo N. Road, Taichung 402, Taiwan
}

\begin{abstract}
The A7 catecholamine cell group consists of noradrenergic (NAergic) neurons that project to the dorsal horn of the spinal cord. Here, we characterized their morphology and physiology properties and tested the effect of substance $P$ (Sub-P) on them, since the results of many morphological studies suggest that $A 7$ neurons are densely innervated by Sub-P-releasing terminals from nuclei involved in the descending inhibitory system, such as the lateral hypothalamus and periaqueductal gray area. Whole cell recordings were made from neurons located $\sim 200 \mu \mathrm{m}$ rostral to the trigeminal motor nucleus (the presumed A7 area) in sagittal brainstem slices from rats aged 7-10 days. After recording, the neurons were injected with biocytin and immunostained with antibody against dopamine- $\beta$-hydroxylase (DBH). DBH-immunoreactive (ir) cells were presumed to be NAergic neurons. They had a large somata diameter $(\sim 20 \mu \mathrm{m})$ and relatively simple dendritic branching patterns. They fired action potentials (AP) spontaneously with or without blockade of synaptic inputs, and had similar properties to those of NAergic neurons in other areas, including the existence of calcium channelmediated APs and a voltage-dependent delay in initiation of the AP (an indicator of the existence of A-type potassium currents) and an ability to be hyperpolarized by norepinephrine. Furthermore, in all DBH-ir neurons tested, Sub-P caused depolarization of the membrane potential and an increase in neuronal firing rate by acting on neurokinin-1 receptors. NonDBH-ir neurons with a smaller somata size were also found in the A7 area. These showed great diversity in firing patterns and about half were depolarized by Sub-P. Morphological examination suggested that the non-DBH-ir neurons form
\end{abstract}

*Corresponding author. Tel: +886-4-24730022x11813; fax: +8864-24757412.

E-mail address: hwy@csmu.edu.tw (H.-W. Yang).

Abbreviations: ACSF, artificial cerebrospinal fluid; AP, action potential; AP5, DL-2-amino-5-phosphonopentanoic acid; A7, A7 catecholamine cell group; DAB, 3,3'-diaminobenzidine; DBH, dopamine- $\beta$-hydroxylase; DNQX, 6,7-dinitroquinoxaline-2,3-dione; HRP, horseradish peroxidase; ir, immunoreactive; LC, locus coeruleus; LH, lateral hypothalamus; mGluR, metabotropic glutamate receptor; Mo5, trigeminal motor nucleus; NAergic, noradrenergic; NE, norepinephrine; NK-1, neurokinin-1; PAG, periaqueductal gray area; PB, phosphate buffer; $\mathrm{Rh}$, rheobase; $\mathrm{Rn}$, neuronal input resistance; $\mathrm{RT}$, room temperature; RVM, rostroventromedial medulla; SD, Sprague-Dawley; SM-Sub-P, $\left[\right.$ Sar ${ }^{9}$, Met $\left.\left(\mathrm{O}_{2}\right)^{11}\right]$-substance $\mathrm{P}$; Sub-P, substance $\mathrm{P}$; TH, tyrosine hydroxylase; TPBS, $0.3 \%$ Triton X-100 in phosphate-buffered saline; TRP, transient receptor potential; TTX, tetrodotoxin; Vm, resting membrane potential; $\tau$, membrane time constant. contacts with DBH-ir neurons. These results provide the first description of the intrinsic regulation of membrane properties of, and the excitatory effect of Sub-P on, A7 area neurons, which play an important role in pain regulation. (c) 2008 Published by Elsevier Ltd on behalf of IBRO.

Key words: antinociception, locus coeruleus, noradrenergic, norepinephrine, NK-1 receptor, pain.

Norepinephrine (NE), one of the most important neuronal modulators in the brain, is known to be involved in the regulation of many brain functions (Aston-Jones and Cohen, 2005), including pain modulation (Pertovaara, 2006). Activation of $\alpha-2$ receptors in the dorsal spinal cord by intrathecal injection of NE or $\alpha-2$ receptor agonists results in dramatic analgesia in rats (Reddy and Yaksh, 1980; Kuraishi et al., 1985; Danzebrink and Gebhart, 1990; Takano and Yaksh, 1992). However, there are no noradrenergic (NAergic) neurons in the dorsal horn of the spinal cord, and it is suggested that the principal sources of NAergic innervation are the locus coeruleus (LC) (also referred to as the $A 6$ cell group) and the $A 7$ catecholamine cell group (A7) area, located, respectively, in the dorsomedial and dorsolateral pons (Westlund and Coulter, 1980; Clark and Proudfit, 1991a; Jones, 1991; Proudfit and Clark, 1991; Kwiat and Basbaum, 1992). Direct stimulation of the A7 area results in an antinociceptive effect which is blocked by intrathecal injection of $\alpha-2$ receptor antagonists, showing that this area plays an important role in intrinsic analgesia mediated by NE acting on $\alpha-2$ receptors at the spinal cord level (Burnett and Gebhart, 1991; Yeomans et al., 1992; Holden et al., 1999).

The intrinsic NAergic pain regulatory system in the spinal cord has only low tonic activity (Dennis et al., 1980), suggesting synaptic drives are needed for activation of this system. One possible source of synaptic drives for A7 cell group activation is the collateral branches of the spinothalamic tract. It has been shown that peripheral noxious stimuli can enhance NE release in the dorsal spinal cord (Takagi et al., 1979; Tyce and Yaksh, 1981), suggesting the existence of reciprocal connections between the $A 7$ cell group and the collateral branches of the ascending spinothalamic tracts that might form a negative feedback system that suppresses the ascending pain signal by causing NE release at the spinal level. NAergic neurons in the A7 area also receive innervation from many of the descending endogenous analgesia systems, including fibers from the lateral hypothalamus (LH), periaqueductal gray area (PAG), and rostroventromedial medulla (RVM) (Yeo- 
mans and Proudfit, 1990, 1992; Clark and Proudfit, 1991b; Holden and Proudfit, 1998; Bajic et al., 2001; Holden et al., 2002). The antinociception induced by stimulation of these nuclei is significantly attenuated by intrathecal injection of $\alpha 2$-receptor antagonists, suggesting the involvement of NE in the analgesia produced by stimulation of these descending inhibitory pathways (Jensen and Yaksh, 1986; Aimone et al., 1987; Iwamoto and Marion, 1993; Peng et al., 1996). Taken together, these morphological and in vivo pharmacological studies suggest that the A7 area is an important part of the endogenous analgesia system and that many other components of this system may exert their antinociceptive effect by controlling the excitability of NAergic neurons in the A7 area, thus changing NE levels in the dorsal spinal cord and modulating nociceptive afferents.

In spite of their important role in modulating nociceptive afferents in the CNS, the intrinsic properties and regulation of the neurons and the local neuronal circuit in the A7 area have not been systemically investigated. It has been shown that substance $P$ (Sub-P) is one of the principal neurotransmitters released from axonal terminals of the $\mathrm{LH}, \mathrm{PAG}$, and RVM into the A7 catecholamine cell group (Holden and Proudfit, 1998; Bajic et al., 2001; Holden et al., 2002); however, physiological evidence for the effect of Sub-P on neurons in the A7 area is limited. In the present study, we first made whole cell recordings from neurons in the A7 area and characterized their firing and membrane properties, then examined the effect of Sub-P on them. Some of the preliminary results have been presented in abstract form (Min et al., 2004).

\section{EXPERIMENTAL PROCEDURES}

\section{Animals and i.m. injection of True Blue for motoneuron labeling}

The use of animals in this study was approved by the Ethical Committee for Animal Research of the National Taiwan University. Every effort was made to minimize the number of animals used and their suffering. Sprague-Dawley (SD) rat pups of both sexes, aged 7-10 days, were used.

Three rat pups were used for trigeminal motor nucleus (Mo5) labeling. They were anesthetized with $5 \%$ isoflurane, then $3 \%$ True Blue (Sigma, USA) in saline was injected into the masseter muscle. After approximately $24 \mathrm{~h}$, brainstem slices were prepared and fixed and immunohistochemical studies performed as described below.

\section{Preparation of brainstem slices}

The preparation of brainstem slices in this study was very similar to that described previously (Min et al., 2003). Briefly, SD rat pups were anesthetized with isoflurane and decapitated. The brain was rapidly exposed and chilled with ice-cold artificial cerebrospinal fluid (ACSF), then sagittal brainstem slices (300 $\mu \mathrm{m}$ thick), comprising the A7 area, Mo5 and surrounding regions, were cut with a vibroslicer (Campden, Loughborough, UK). In most cases, only one slice was obtained from one hemisphere of brainstem. The ACSF contained (in mM): $\mathrm{NaCl} 105, \mathrm{KCl} 5, \mathrm{MgSO}_{4} 1.3, \mathrm{NaHCO}_{3}$ 24, $\mathrm{NaH}_{2} \mathrm{PO}_{4} 1.2, \mathrm{CaCl}_{2}$ 2, and glucose 10, with the $\mathrm{pH}$ adjusted to 7.4 by gassing with $95 \% \mathrm{O}_{2} / 5 \% \mathrm{CO}_{2}$. The slices were kept in an interface-type chamber at room temperature (RT) $\left(24-25^{\circ} \mathrm{C}\right)$ for at least 90 min to allow recovery.

\section{Visualization of neurons in the A7 area and whole cell patch clamp recording}

Slices were transferred to an immersion-type recording chamber mounted on an upright microscope (BX51WI, Olympus Optical Co., Ltd., Tokyo, Japan), equipped with a water-immersion objective $(40 \times)$ and a Normaski optic system. Recordings were made from neurons located about $200 \mu \mathrm{m}$ rostral to the anterior border of Mo5 with patch pipettes, using procedures described by Stuart et al. (1993). Unless otherwise specified, only one neuron was recorded per slice. The patch pipettes were pulled from borosilicate glass tubing $(1.5 \mathrm{~mm}$ outer diameter, $0.5 \mathrm{~mm}$ wall thickness; Warner Instruments Corporation, Hamden, CT, USA), and had a resistance of about $3 \mathrm{M} \Omega$ when filled with internal solution. The internal solution contained (in $\mathrm{mM}$ ): potassium gluconate 131, EGTA 2, $\mathrm{MgCl}_{2}$ 5, Hepes 40, ATP 3; GTP 0.3 and biocytin 10, with the $\mathrm{pH}$ adjusted to 7.2 with $\mathrm{KOH}$ and the osmolarity to 300 mOsm. All recordings were made at RT $\left(25-27^{\circ} \mathrm{C}\right)$. Once whole cell recordings were obtained, the patch amplifiers (Axopatch 1D; Axon Instruments Inc.; Union City, CA, USA) were set to current clamp mode and the bridge was balanced by adjusting the serial resistance compensation of the amplifiers. Neurons were only accepted for further study if the membrane potential $(\mathrm{Vm})$ was at least $-45 \mathrm{mV}$ without applying a holding current and the action potential (AP) was able to overshoot $0 \mathrm{mV}$. Signals were low-pass filtered at a corner frequency of $2 \mathrm{kHz}$ and digitized at $10 \mathrm{kHz}$ using a Micro 1401 interface running Signal and Spike2 software provided by Cambridge Electronic Design (Cambridge, UK).

\section{Filling of recorded neurons with biocytin and immunohistochemistry}

Neurons were filled by passive diffusion of biocytin from the patch pipette during the recording period. After recording, the pipettes were withdrawn and the slices left in the recording chamber for 30 min to allow biocytin transport within the dendrites and axon. The brain slices were then transferred to $4 \%$ paraformaldehyde (Merck, Frankfurt, Germany) in $0.1 \mathrm{M}$ phosphate buffer (PB), pH 7.4 , for fixation overnight at $4{ }^{\circ} \mathrm{C}$, then were subjected to histological procedures for visualization of biocytin-filled neurons without further sectioning. The slices were incubated for $1 \mathrm{~h}$ at RT in $0.3 \%$ Triton X-100 in phosphate-buffered saline (TPBS) containing $10 \%$ normal goat serum and $2 \%$ bovine serum albumin, then overnight at $4{ }^{\circ} \mathrm{C}$ with a mixture of AMCA-avidin $\mathrm{D}$ (dilution:1/250; Vector Laboratories, Burlingame, CA, USA) and monoclonal mouse antibody against rat dopamine- $\beta$-hydroxylase (DBH) (dilution: 1/1300; Chemicon, Temecula, CA, USA) in TPBS. In the experiments in Fig. 1, polyclonal rabbit antibody against rat tyrosine hydroxylase (TH) (dilution: 1/1000; Chemicon, Temecula, CA, USA) was also included. The slices were then incubated for $2 \mathrm{~h}$ at RT with tetramethyl rhodamine-conjugated horse antibody against mouse IgG (Jackson, ImmunoResearch Lab. Inc., West Grove, PA, USA). In the experiments in Fig. 1, fluorescein isothiocyanate (FITC)-conjugated goat antibody against rabbit IgG (Jackson, ImmunoResearch Lab, Inc., PA, USA) was also included in the incubation solution. The slices were washed at least three times (10 min per wash) with TPBS between incubation steps. Biocytin-filled neurons were examined for binding of anti$\mathrm{DBH}$ and TH antibodies and photographed on an Olympus BX-50 fluorescent microscope (Olympus Optical Co., Ltd.).

\section{Analysis of the membrane properties and electrophysiological data}

Only neurons confirmed as being within the A7 area (located about 200-300 $\mu \mathrm{m}$ rostral to Mo5 and surrounded by a cluster of $\mathrm{DBH}$-immunoreactive (ir) neurons) (see Fig. 1) were used for further physiological and morphological analysis. Once a whole cell recording was obtained, the resting membrane potential $(\mathrm{Vm})$ 

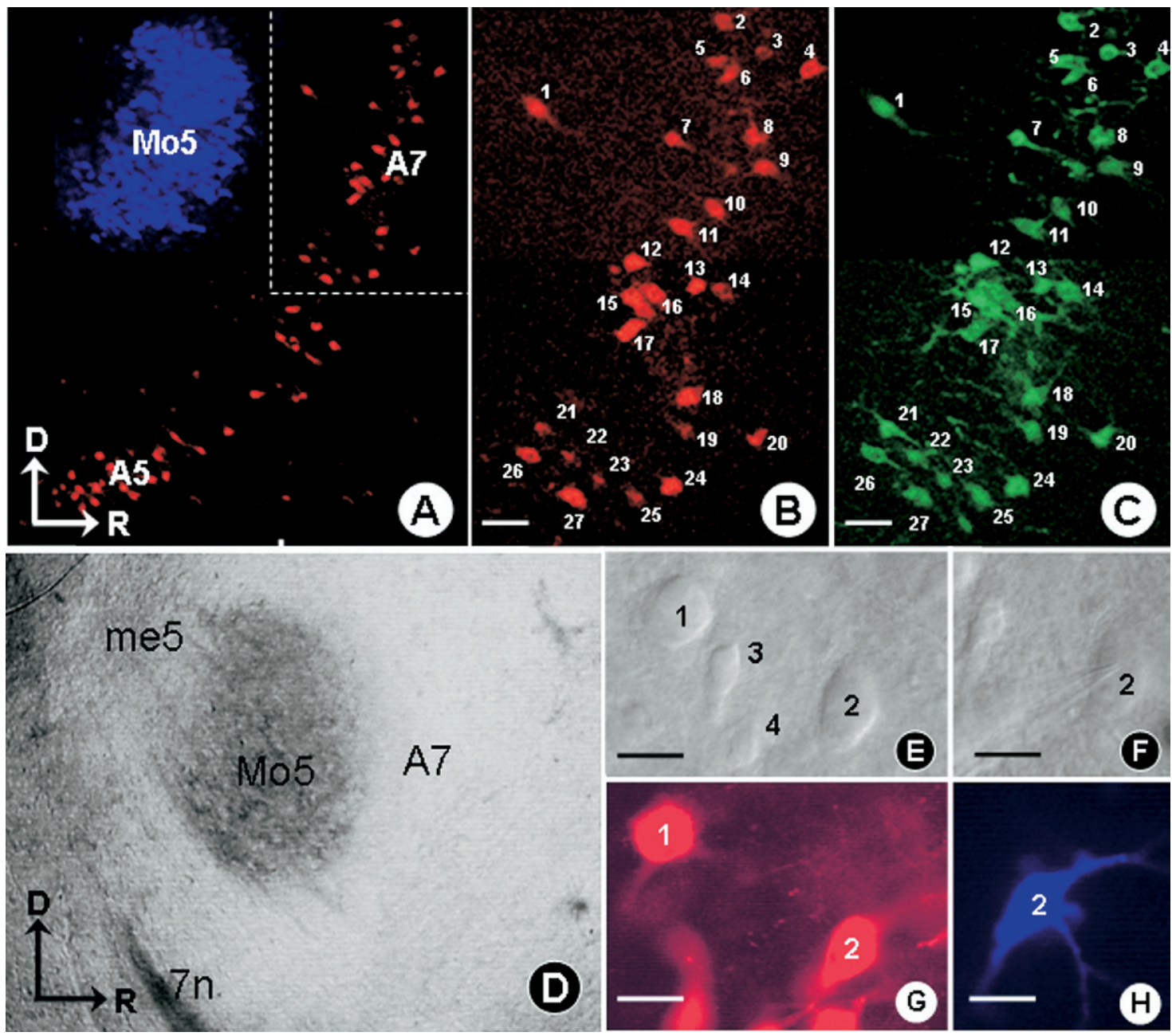

Fig. 1. Neuronal cell types in the A7 area. (A) Merged images of Mo5 labeled by i.m. injection of True Blue (blue) and cells immunostained with anti-DBH antibodies (red: TRITC) considered to be the A7 and A5 catecholamine cell groups. Note that the A5 and A7 areas are, respectively, ventral and rostral to Mo5. The horizontal and vertical arrows indicate the rostral (R) and caudal (D) orientation of the slice. The length of the arrows also serves as the scale, which is $125 \mu \mathrm{m}$. (B) High-power micrograph of the A7 area (dotted square in A) showing 27 DBH-ir neurons. (C) High-power micrograph of the same area stained with anti-TH antibodies (labeled with FITC). Note that all 27 DBH-ir neurons shown in B are also TH-ir, suggesting they are all NAergic. (D) IR-DIC image of a living brain slice showing the relative locations of Mo5 and the presumed A7 area at low power. Neighboring structures, such as the 7th nerve (7n) and mesencephalic 5 tract (me5), are also labeled. The horizontal and vertical arrows show the rostral (R) and caudal (D) orientation of the slice, and their lengths also serve as the scale, which is $125 \mu \mathrm{m}$. (E, F) IR-DIC images of the presumed A7 area shown at high power. Note the existence of large (cells 1 and 2) and small (cells 3 and 4) neurons in this area, with a large neuron (cell 2) being recorded $(F)$ and injected with biocytin. (G, H) DBH immunostaining (G) and biocytin-avidin-D-AMCA (H) staining after recording, showing that the recorded neuron (cell 2), labeled with AMCA is DBH-ir. The other large neuron (cell 1) seen in the IR-DIC image is DBH-ir, while the two small neurons (cells 3 and 4 ) are not. Scale bars $=50 \mu \mathrm{m}(\mathrm{B}, \mathrm{C}) ; 20 \mu \mathrm{m}(\mathrm{E}-\mathrm{H})$.

was determined, then a small amount of hyperpolarizing current was injected to hold the $\mathrm{Vm}$ at $\sim-70 \mathrm{mV}$ and stop spontaneous firing. The neuronal input resistance $(\mathrm{Rn})$ was taken as the slope of the linear portion of the current-voltage relationship plot (see asterisks in online Supplemental Fig. 1A2), constructed from the transmembrane responses to different current injection. The amplitude of the AP was taken as the voltage difference from $\mathrm{Vm}$ to the highest point of the AP evoked by depolarizing current pulses, and the minimum current intensity required to evoke an AP was taken as the rheobase (Rh) (online Supplemental Fig. 1B). The half-width (HW) of the AP was taken as the time interval between the rising and falling phases of the AP at the level of the halfamplitude (online Supplemental Fig. 1B). For the membrane time constant $(\tau)$, an averaged transmembrane response to a $0.5 \mathrm{~ms}$ hyperpolarizing current pulse, given at $500-1000 \mathrm{pA}$, was obtained from at least 1000 sweeps (see inset in online Supple- mental Fig. 1C). The natural logarithm of the decay of this potential with time was plotted, voltage changes within the first $1 \mathrm{~ms}$ of the current pulses being ignored. The linear portion of the plot was determined by eye (see arrows in online Supplemental Fig. 1C), and the best-fit straight line to the intervening data points fitted by the method of least squares using Origin software (see straight line in online Supplemental Fig. 1C). The value of $\tau$ was given by the time taken for the voltage to decay to $1 / \mathrm{e}$ of its initial value.

Current pulses were applied to examine the firing pattern of neurons. Spontaneous firing and the effect of Sub-P and NE were recorded and analyzed using Spike2 software. The effect of Sub-P on the firing rate was estimated as $\left(R_{b}-R_{a}\right) \times 100 \% /\left(R_{b}+R_{a}\right)$, where $R_{a}$ and $R_{b}$ are the firing rates calculated before and during application of Sub-P or [Sar ${ }^{9}$, Met $\left.\left(\mathrm{O}_{2}\right)^{11}\right]$-Substance P (SM-Sub$\mathrm{P})$, a neurokinin-1 (NK-1) receptor agonist. 
Table 1. Membrane and spike properties of neurons in the A7 catecholamine cell group at room temperature

\begin{tabular}{llccccc}
\hline & $\mathrm{Vm}(\mathrm{mV})$ & $\mathrm{Rn}(\mathrm{M} \Omega)$ & $\mathrm{Rh}(\mathrm{pA})$ & $\tau(\mathrm{ms})$ & $\mathrm{Amp}(\mathrm{mV})$ & $\mathrm{HW}(\mathrm{ms})$ \\
\hline DBH-ir & $-55 \pm 2(N=26)$ & $526 \pm 41^{*}(N=26)$ & $103 \pm 4^{*}(N=26)$ & $32 \pm 3^{*}(N=6)$ & $110 \pm 2(N=26)$ & $3.0 \pm 0.1(N=26)$ \\
Non-DBH-ir & $-53 \pm 1(N=22)$ & $1147 \pm 152^{*}(N=22)$ & $52 \pm 4^{*}(N=22)$ & $58 \pm 7^{*}(N=5)$ & $106 \pm 2(N=22)$ & $2.9 \pm 0.2(N=22)$ \\
\hline
\end{tabular}

* $P<0.05$, significant difference using the Mann-Whitney test.

$\mathrm{HW}$, half-width; $\mathrm{R}_{\mathrm{h}}$, minimum amount of current injection required for discharging action potentials; Amp, action potential amplitude.

All chemicals used for ACSF and pipette solution preparation were from Merck; picrotoxin, strychnine, Sub-P and NE were from Sigma (St. Louis, USA); and DL-2-amino-5-phosphonopentanoic acid (AP5), 6,7-dinitroquinoxaline-2,3-dione (DNQX), nimodipine, RP67580, SM-Sub-P and tetrodotoxin (TTX) were from TocrisCookson (Bristol, UK).

\section{Analysis of the morphological data}

In some slices after electrophysiological recording, the following modifications were made to our normal histological procedure for viewing biocytin-filled neurons so that the fine structure of the filled neurons could be revealed for morphological measurement and reconstruction. Firstly, after fixation, the slices were treated with $3 \% \mathrm{H}_{2} \mathrm{O}_{2}$ in $\mathrm{PB}$ for 20 min to quench endogenous peroxidase. Secondly, in addition to AMCA-avidin D and anti-DBH antibody, a small amount of $A B C$ kit solution (dilution: 1/500 Vector Laboratories) was added to the primary antibody cocktail, and the slices incubated overnight at $4{ }^{\circ} \mathrm{C}$ in this cocktail. Both the $A B C$ complex and AMCA-avidin $D$ bind to the biocytin used, so the recorded neurons were labeled with both AMCA and horseradish peroxidase (HRP). Thirdly, following fluorescent microscopy, the slices were incubated overnight at $4{ }^{\circ} \mathrm{C}$ with biotinylated HRP (solution B of the $A B C$ kit; dilution 1/500) to saturate the bound avidin with biotin and maximize the intensity of HRP labeling in the recorded neurons. Fourthly, 3,3'-diaminobenzidine (DAB; Sigma) was used as chromogen; the slices were incubated with a solution of 0.5 $\mathrm{mg} / \mathrm{ml}$ of $\mathrm{DAB}$ and $0.0003 \% \mathrm{H}_{2} \mathrm{O}_{2}$, then were mounted on gelatincoated slides, dehydrated with graded concentrations of alcohol, and covered with DPX for microscopy. The recorded neurons were examined, photographed and reconstructed using an Olympus BX-50 microscope and a camera lucida drawing tube (SZXDA, Olympus Optical Co., Ltd.). The drawings of the reconstructed biocytin-filled neurons were digitized using a PC-based scanner, and measurements made of the soma minimum and maximum diameters, the dendrite segment length and the branching of the axonal collaterals using $\mathrm{NIH}$-Image software (downloaded from the National Institutes of Health website http://www.nih.gov).

All data are expressed as the mean \pm standard error of the mean; the paired-test or Mann-Whitney $U$ test was used for statistical comparisons.

\section{RESULTS}

\section{Recording from neurons in the A7 area}

When sagittal brainstem slices prepared from the brain of rat pups injected with $3 \%$ True Blue in the masseter muscle were stained with antibodies against $\mathrm{DBH}$ and $\mathrm{TH}$, two populations of neurons staining for both $\mathrm{DBH}$ and $\mathrm{TH}$ were identified in the rostral and ventral borders of Mo5 (Fig. $1 \mathrm{~A}-\mathrm{C})$. Since no neurons staining only for $\mathrm{TH}$ were seen, these results indicated that these two groups of neurons were mostly NAergic and not dopaminergic. They were presumed to be, respectively, the A7 and A5 cell groups, located rostrally and ventrally to Mo5 (Paxinos and Watson, 1998; Min et al., 2007). In living slices, using IR-DIC micros- copy video, two types of neurons could be identified in the A7 area by the diameter of their somata (Fig. 1D-F). Immunostaining showed that the neurons with the larger somata diameter were $\mathrm{DBH}$-ir, whereas those with the smaller somata diameter were not (Fig. 1E-H; Fig. 6). These results show the existence of large NAergic principal neurons and small non-NAergic interneurons in the A7 area.

\section{Physiological properties of the non-NAergic neurons in the A7 area}

Membrane and spike property measurements were made from 26 NAergic neurons and 22 non-NAergic interneurons in 46 slices cut from 27 animals; the results are summarized in Table 1. No significant differences were found between the two groups of neurons in most of the parameters examined, except for $\mathrm{Rn}, \mathrm{Rh}$ and $\tau$ (Mann-Whitney $U$ test). The NAergic neurons had a significantly smaller $\mathrm{Rn}$, higher $\mathrm{Rh}$ and shorter $\tau$ than the non-NAergic interneurons. These results are consistent with the morphological measurements, which showed that the averaged somata diameter, defined as (maximum + minimum diameter)/2, of the NAergic neurons was $\sim 1.7$ times greater than that of the non-NAergic interneurons $(22 \pm 0.7 \mu \mathrm{m}$ vs. $13 \pm 2.1 \mu \mathrm{m} ; P<0.01$, Mann-Whitney $U$ test).

Since previous in vitro studies on NAergic neurons in the LC and subcoeruleus nuclei (Williams et al., 1984; Brown et al., 2006) used a more physiological temperature of around $35{ }^{\circ} \mathrm{C}$, it was important to consider possible effects of recording temperature (RT vs. $35{ }^{\circ} \mathrm{C}$ ) when comparing the present data with those from previous studies. To address the possible effect of recording temperature (RT vs. $35^{\circ} \mathrm{C}$ ) on membrane and firing properties of A7 neurons, another $12 \mathrm{DBH}$-ir neurons from six slices were recorded at $35{ }^{\circ} \mathrm{C}$ (Table 2) and the results compared. Membrane properties, such as the Rn and Rh, and the rate of spontaneous firing of $A 7$ neurons, were significantly different from those recorded at RT; these differences can be attributed to the overall higher enzyme and transporter activity and slight increase in soma diameter (averaged somata diameter: $25 \pm 0.4 \mathrm{~mm}$ ).

A further 37 non-NAergic interneurons obtained from 12 slices from six animals were recorded for firing pattern analysis. They showed a large diversity in firing pattern and could be grouped into five categories based on the two criteria of whether they showed spontaneous firing and/or showed voltage sag/rebound AP in response to injection of a hyperpolarizing current pulse (Fig. 2F). Six of the 37 showed both voltage sag/rebound AP (see arrow and asterisk in Fig. 2A) and spontaneous firing, and were classified as Type A interneurons (Fig. 2A). Another 15 also 
Table 2. Membrane and spike properties of neurons in the $\mathrm{A} 7$ catecholamine cell group at $35^{\circ} \mathrm{C}$

\begin{tabular}{|c|c|c|c|c|c|c|}
\hline & $\mathrm{Vm}(\mathrm{mV})$ & $\mathrm{Rn}(\mathrm{M} \Omega)$ & $\mathrm{Rh}(\mathrm{pA})$ & $\tau(\mathrm{ms})$ & $A m p(m V)$ & $\mathrm{HW}(\mathrm{ms})$ \\
\hline DBH-ir & $-58 \pm 3(N=12)$ & $274 \pm 16^{*}(N=12)$ & $99 \pm 3^{*}(N=26)$ & $63 \pm 4^{*}(N=9)$ & $99 \pm 3^{*}(N=26)$ & $1.4 \pm 0.1^{*}(N=12)$ \\
\hline
\end{tabular}

showed sag/rebound AP, but did not fire spontaneously, and six of these showed spike adaptation in response to depolarizing current injection (see arrowhead in Fig. 2B); those showing spike adaptation were classified as Type B interneurons (Fig. 2B) and those without spike adaptation were classified as Type $C$ interneurons (Fig. 2C). The remained 16 neurons did not show voltage sag/rebound-AP; 3 showed spontaneous firing and were classified as Type D (Fig. 2D), while the other 13 did not and were classified as Type $E$ interneurons (Fig. 2E). No significant difference was found in $\mathrm{Rn}, \mathrm{Rh}$ and $\tau$ between these different groups of non-NAergic interneurons (Mann-Whitney $U$ test).

\section{Physiological properties of the NAergic neurons in the $A 7$ area}

The firing pattern of the NAergic neurons was highly constant. More than half of the recorded NAergic neurons fired APs spontaneously. The mean firing rate for nine neurons in normal ACSF was $0.28 \pm 0.11 \mathrm{~Hz}$. In some neurons, burst firing was noted in some periods of recording (Fig. $3 \mathrm{~A})$. When a drug cocktail containing $10 \mu \mathrm{M}$ DNQX, $50 \mu \mathrm{M}$ AP5, $100 \mu \mathrm{M}$ picrotoxin and $1 \mu \mathrm{M}$ strychnine was added to the ACSF to completely block both excitatory and inhibitory synaptic transmission, the mean firing rate for 16 neurons was increased to $0.53 \pm 0.09 \mathrm{~Hz}$, suggesting that the intrinsic membrane properties of the NAergic neurons in the A7 area allow spontaneous firing, which is modulated by synaptic inputs on to these neurons. In response to injection of a hyperpolarizing current pulse of high intensity, the NAergic neurons showed no voltage sag/rebound AP (see online Supplemental Fig. 1A). When the Vm was held at $\sim-75 \mathrm{mV}$, two obvious features seen in all NAergic neurons recorded were the appearance of a marked delay in the initiation of an AP upon injection of a depolarizing current pulse, and, once excited, the firing of a train of APs with a regular interval and without any sign of AP adaptation (Fig. 3B). When a depolarizing current pulse of long duration was injected into the neurons to increase the firing rate, a delay in AP initiation was also seen after termination of a further applied hyperpolarizing current pulse (Fig. $3 \mathrm{C} 1$ ). It is notable that the delay time of AP initiation was highly voltage-dependent (Fig. 3C2).

\section{Morphological properties of the neurons in the A7 area}

In order to obtain better images clearly showing the morphology of the recorded neurons, after immunofluorescent labeling and fluorescence microscopy, we incubated the slices with HRP-conjugated biotin, then detected bound HRP using DAB as chromogen. Fig. 4 shows two exam- ples of the resultant staining, which clearly revealed the dendritic arbors, axonal trajectory and somatic morphology of the NAergic neurons. Quantitative morphological data were obtained from 10 NAergic neurons with a total of 44 dendrites. The NAergic neurons had an averaged number of $4.7 \pm 0.4$ primary dendrites (range 2 to 6 ). On average, these dendrites branched $2 \pm 0.2$ times (range 0 to 5 ), and the averaged lengths of the dendritic segment and dendritic path were $101 \pm 6$ and $214 \pm 9 \mu \mathrm{m}$, respectively (range 16-310 $\mu \mathrm{m}$ and $44-526 \mu \mathrm{m}$, respectively). Axonal arborization was also clearly identified in some of the analyzed neurons, including the two examples shown in Fig. 4 (in red). In general, the axons of the NAergic neurons ran dorsally and caudally toward the medulla (Fig. 4). In all cases in which the axon was identified, a number of varicosities could be clearly identified in the $A 7$ area (see insets a5 and b3 in Fig. 4). In three cases, axonal terminals were also identified in Mo5, indicating that the NAergic neurons of the $A 7$ catecholamine cell group also projected to Mo5 (see Min et al., 2007).

The presence of varicosities of NAergic neurons in the A7 area suggested the possibility of autoregulation of NAergic neurons. We confirmed this possibility by showing that application of $10 \mu \mathrm{M} \mathrm{NE}$ resulted in hyperpolarization of the $\mathrm{Vm}$ in all 18 NAergic neurons tested (Fig. $5 A$ and $B$ ). The mean amplitude of hyperpolarization by $10 \mu \mathrm{M}$ NE was $14.1 \pm 1.6$ $\mathrm{mV}$ (Fig. 5C). In the 10 neurons that showed spontaneous firing, the firing rate was almost completely suppressed by NE (Fig. 5A and D). The mean firing rate for these 10 neurons was $0.62 \pm 0.15 \mathrm{~Hz}$, which was decreased to $0.04 \pm 00.03 \mathrm{~Hz}$ by NE $(P<0.00 \mathrm{l}$; paired $t$-test) and recovered to $0.64 \pm 0.2 \mathrm{~Hz}$ after washout (Fig. 5D). The effect of NE on the Rn was tested in six NAergic neurons; the $\mathrm{Rn}$ was $509 \pm 57 \mathrm{M} \Omega$ in control conditions, decreased to $362 \pm 32 \mathrm{M} \Omega$ in the presence of $\mathrm{NE}(P<0.001$, paired $t$-test) and recovered to $477 \pm 59 \mathrm{M} \Omega$ after washout (Fig. 5B and E). NE also reduced the excitability of NAergic neurons, because they stopped firing even when the Vm was held at the resting level, as in the control conditions (compare traces $\mathrm{i}$, ii, and iv in Fig. 5B).

In some experiments, both NAergic neurons and nonNAergic interneurons in the same slice were recorded and filled with biocytin (Fig. 6), allowing us to examine morphologically possible interactions between these two types of neurons. In the 22 pairs of NAergic and non-NAergic interneurons examined, contacts of the axon of the nonNAergic interneurons on the dendrites or soma of the NAergic neurons were found in 14 cases; Fig. 6 shows a typical example. A pair of neurons, one larger than the other, was recorded and labeled (inset a). DBH immunostaining confirmed that only the large neuron was $\mathrm{DBH}$-ir (insets $b, c$ ), and subsequent HRP staining using DAB as 
A
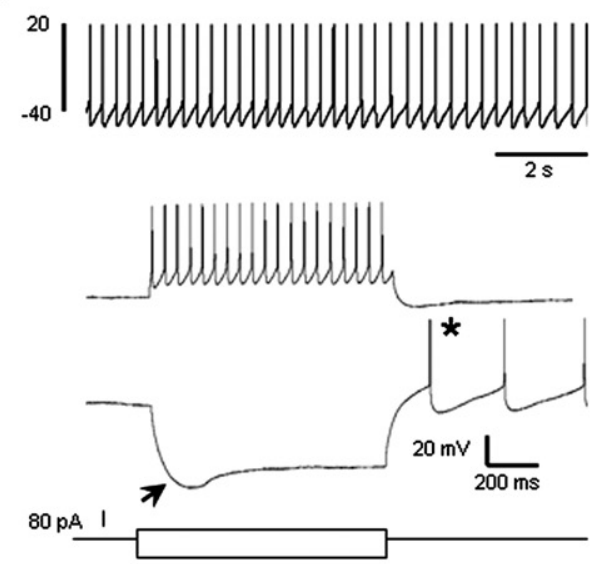

C

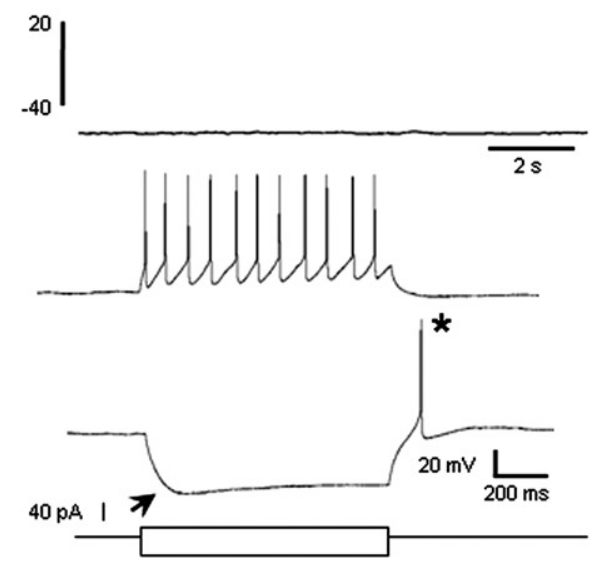

E

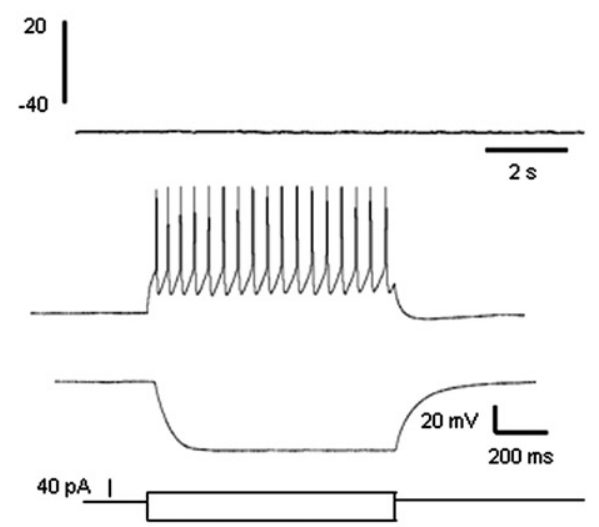

B

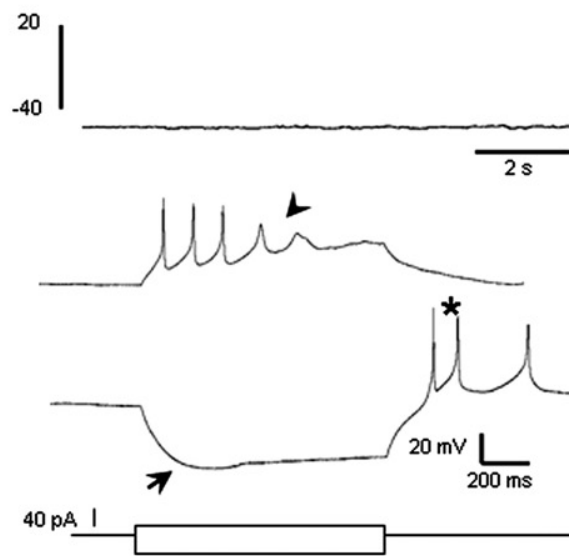

D
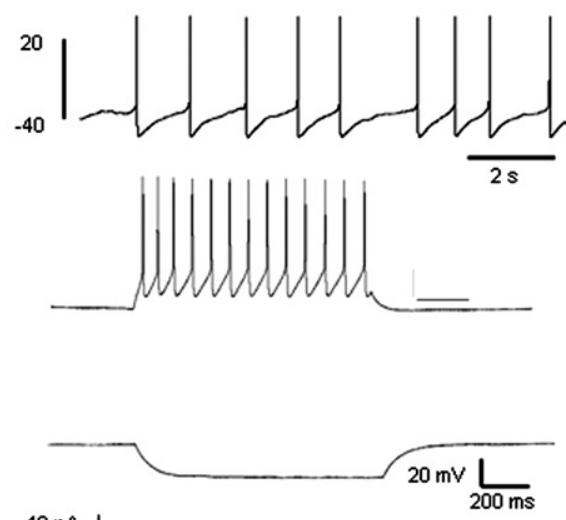

$40 \mathrm{pA}$ I

F

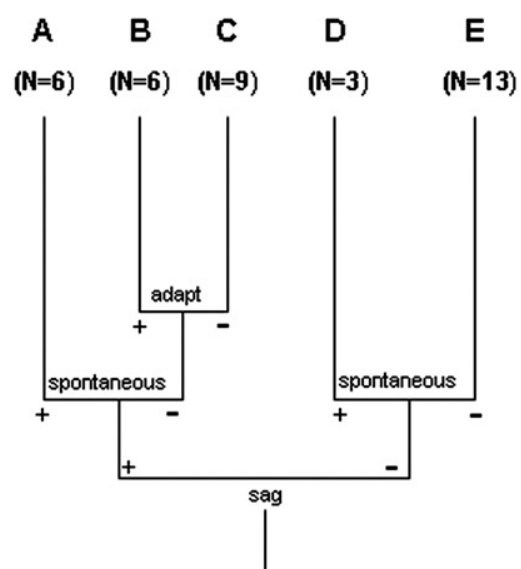

Fig. 2. Firing patterns of non-NAergic interneurons. (A-E) Typical examples of the firing properties of each type of interneuron in the A7 area. In each panel, the top trace is a long period of recording without any current injection or drug application, the middle two traces are the voltage responses to depolarizing and hyperpolarizing current injection, and the bottom section shows the polarity and intensity of the injected current. Note that only types $A$ and $D$ show spontaneous firing. Types $A, B$ and $C$ show voltage sag and rebound AP, indicated by an arrow and asterisk, respectively. Type B also shows spike adaptation in response to depolarizing current injection, as indicated by the arrowhead. (F) Diagram summarizing the firing characteristics of the different types of non-NAergic interneurons.

chromogen revealed the entire morphology of the two neurons (inset $d$ ). The dotted square in the reconstruction shows a dendrite of the NAergic neuron (arrowheads in inset e) running parallel to an axonal branch of the non-NAergic interneuron (arrows in e), with physical contacts between the two neurons (white circles in e). These results suggest possible synaptic connections between the NAergic neurons and non-NAergic interneurons. 
A
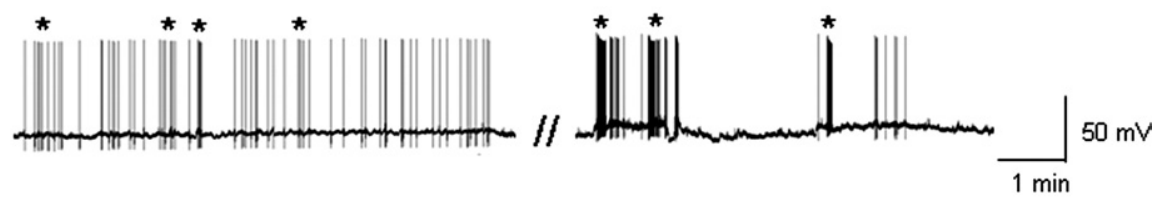

B1

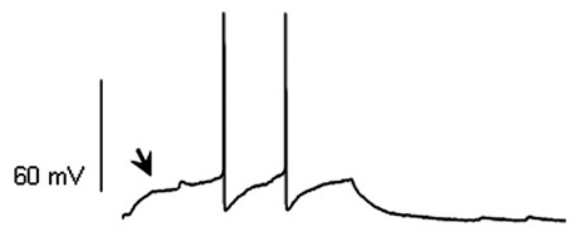

B2

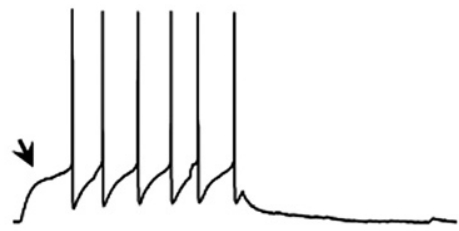

B3
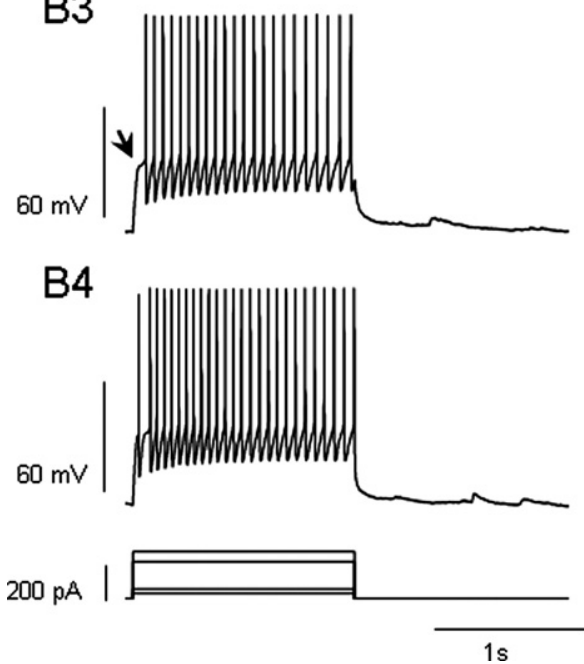

C1

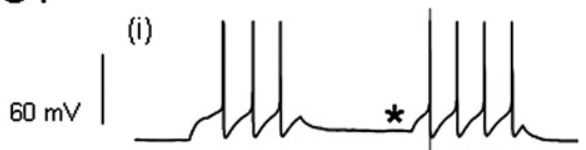

(ii)<smiles>[18OH][18OH]</smiles>

(iii)

$60 \mathrm{mV}$

$50 \mathrm{pA} \mid$

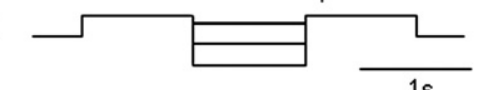

C2

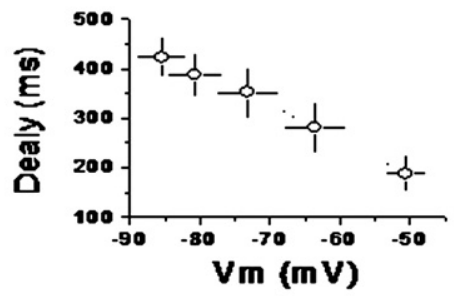

Fig. 3. Firing properties of NAergic neurons. (A) Recording of spontaneous firing activity of a NAergic neuron. Note the appearance of burst firing marked by asterisks, which was sometimes very prominent, as shown on the right trace, a recording made 60 min after the activity shown on the left. (B) Membrane voltage responses to different intensities of depolarizing current injection (shown in the bottom panel) in another NAergic neuron. Note the delay in initiation of the AP (arrows in B1-B3). Initiation of the AP without delay was only seen when a high intensity current was injected (B4). (C) Voltage response of a NAergic neuron to injection of a long depolarizing current pulse (50 pA) plus shorter hyperpolarizing current pulses of different intensity superimposed in the middle of the depolarizing current pulse $(\mathrm{Cl}$, traces $\mathrm{i}$-iii; the lowest trace shows the paradigm of current injection). Note the delay in AP initiation after hyperpolarizing current injection (indicated by the dotted line), and that the higher the intensity of the current injected, the longer the delay time. C2 shows results from six cells as a plot of Vm during hyperpolarizing current injection (indicated by the asterisks in C1) versus delay time, showing the voltage-dependence of the AP delay.

\section{Effect of Sub-P on neurons in the A7 area}

We then tested the responses of neurons in the A7 area to bath application of Sub-P. Depolarization of the $V m$ and an increase in firing rate were observed in all six NAergic neurons tested under blockage of synaptic inputs; a typical example is shown in Fig. 7A. The mean firing rate was increased from $0.52 \pm 0.26$ to $2.34 \pm 0.18 \mathrm{~Hz}$ ( $n=$ six cells; $P<0.005$, paired t test) by $0.2 \mu \mathrm{M}$ Sub-P. The percentage change in the firing rate was calculated (see Experimental Procedures) and used to create a dose-response curve
(Fig. 7B). The estimated $\mathrm{EC}_{50}$ for the effect of Sub-P was $18 \mathrm{nM}$. The effects of $0.2 \mu \mathrm{M}$ Sub-P were mimicked by $0.2 \mu \mathrm{M}$ SM-Sub-P, an NK-1 receptor agonist, which increased the firing rate of the NAergic neurons from $0.46 \pm 0.33 \mathrm{~Hz}$ to $3.22 \pm 0.29 \mathrm{~Hz}(n=7$ cells, $P<0.005$, paired $t$-test); a typical example is shown in Fig. $7 \mathrm{C}$. The effect of SM-Sub-P was significantly attenuated by $10 \mu \mathrm{M}$ RP67580, an NK-1 receptor blocker (Fig. 7D). When slices were first perfused with RP67580 for more than $1 \mathrm{~h}$, the effect of SM-Sub-P was completely blocked in two of three neurons 


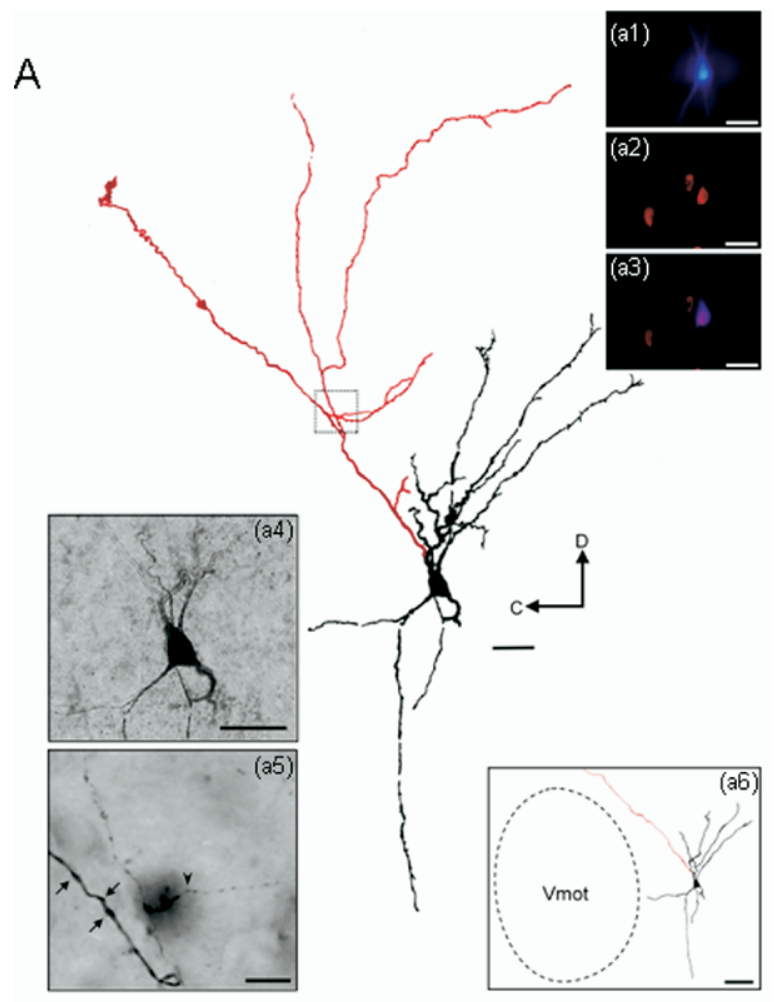

B

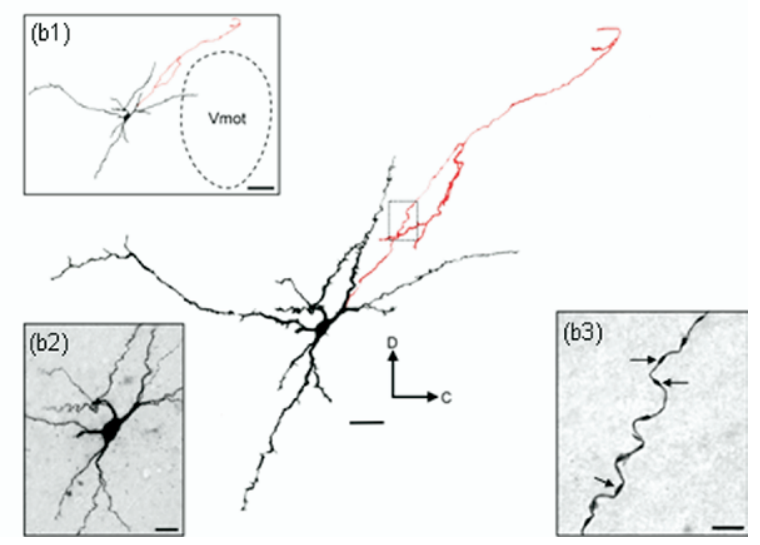

Fig. 4. Morphology of NAergic neurons. (A, B) Camera lucida drawing showing the morphology of two NAergic neurons, with the soma and dendrites in black and the axonal arbors in red. Insets a1-a3 show immunostaining results confirming the reconstructed cell is $\mathrm{DBH}$-ir (a1: biocytin filling; a2: DBH immunostaining; a3: merged image). Insets a4 and $b 2$ show photographs of the same recorded neurons stained with $D A B$ as chromogen. Note the fine structure of the filled neurons, such as the axonal varicosities, shown in insets a5 and b3 (arrows). The location of these axonal varicosities is indicated by the dotted square in the camera lucida drawing, which is near the soma of the recorded neuron. Insets $\mathrm{a} 6$ and $\mathrm{b} 1$ are camera lucida drawings at low power to indicate the spatial relationship between the recorded NAergic neurons and Mo5. Note the axons run dorsocaudally in both cases. Scale bars $=50 \mu \mathrm{m}$ (main reconstruction in A, B and a1-a4); $10 \mu \mathrm{m}$ (a5, b3); $100 \mu \mathrm{m}$ (a6, b1); $25 \mu \mathrm{m}$ (b2).

tested. To reduce the time of exposure of slices to DMSO (vehicle for RP67580), in another seven experiments, RP67580 was added to the ACSF only 10-15 min before application of SM-Sub-P. In this case, RP67580 partially blocked (55.7\% of control levels) the effect of SM-Sub-P, as $0.2 \mu \mathrm{M}$ SM-Sub-P still increased the firing rate of NAergic neurons from $0.48 \pm 0.38 \mathrm{~Hz}$ to $1.18 \pm 0.34 \mathrm{~Hz}$ ( $n=7$ cells, $P=0.067$, paired $t$-test). This might be due to the fact that RP67580 is hydrophobic and $10-15 \mathrm{~min}$ is not sufficient for it to penetrate the slice. However, when expressed as the percentage change in firing rate, the effect of SM-Sub-P on the firing rate after 15 min treatment with RP67580 (41.8 $17.1 \%$ increase, $n=7$ ) was significantly less than the effect in the control $(75 \pm 8 \%$ increase, $n=7 ; P<0.05$; Mann-Whitney $U$ test). Fig. 7E shows the summarized results for the effect of Sub-P and SM-Sub-P on the mean firing frequency of NAergic neurons and the attenuating effect of RP67580 (pooling the data for $1 \mathrm{~h}$ and 15 min perfusion). The above results showed that the Sub-P was acting on NK-1 receptors. The increase in the neuronal firing rate caused by Sub-P was accompanied by a slight decrease in the Rn (Fig. 7F), although the effect was not significant $(95.2 \pm 4.1 \%$ of control; $n=5, P=0.058$, paired $t$-test).

Sub-P caused membrane depolarization in some, but not all, non-NAergic interneurons. It caused membrane depolarization in two of six type $A$ interneurons, one of six type $B$ interneurons, four of nine type $C$ interneurons, one of three type $D$ interneurons and 7 of 13 type $E$ interneurons tested. Fig. 8 shows typical example of recordings made in two type $C$ (Fig. 8A) and two type $E$ (Fig. 8B) interneurons, in each case with one responding and one not responding to Sub-P.

\section{DISCUSSION}

In this study, we provide the first description of the electrophysiological and morphological properties of neurons of the A7 catecholamine cell group and of the possible autoregulation of the NAergic neurons. We also provide evidence for a role of Sub-P, acting at NK-1 receptors, in modulating the $V m$ and firing rate of $A 7$ neurons, results consistent with those of morphological studies showing that $A 7$ neurons were innervated by a large number of Sub-P-ir axonal terminals from the RVM, PAG and LH. In addition, many types of presumed interneurons displaying different physiological properties were found in the A7 area. Interestingly, the axonal terminals of these presumed interneurons were found to make contacts with $\mathrm{DBH}$-ir neurons, showing that there are intimate interactions between non-NAergic interneurons and NAergic neurons in the $A 7$ area.

\section{Neuron identification}

In the present study, all neurons included in data analyses were located rostral to Mo5 (see Min et al., 2007). In rats, according to Paxinos and Watson (1998) and many other authors (Dahlström and Fuxe, 1964; Westlund and Coulter, 1980; Kwiat and Basbaum, 1990), this region corresponds to the A7 catecholamine cell group; it is also called the Kölliker-Fuse nucleus (Nag and Mokha, 2004). Since the A7 area is close to Mo5 and more than $500 \mu \mathrm{m}$ 
A $10 \mu \mathrm{MNE}$

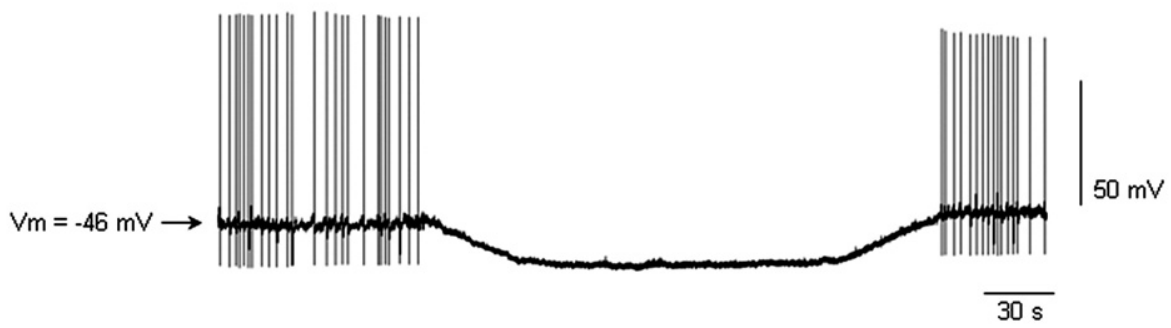

B
(i) Control
(ii) NE
(iii) $\mathrm{NE}+l_{\text {hold }}$ on
(iv) Wash $+l_{\text {hold }}$ off

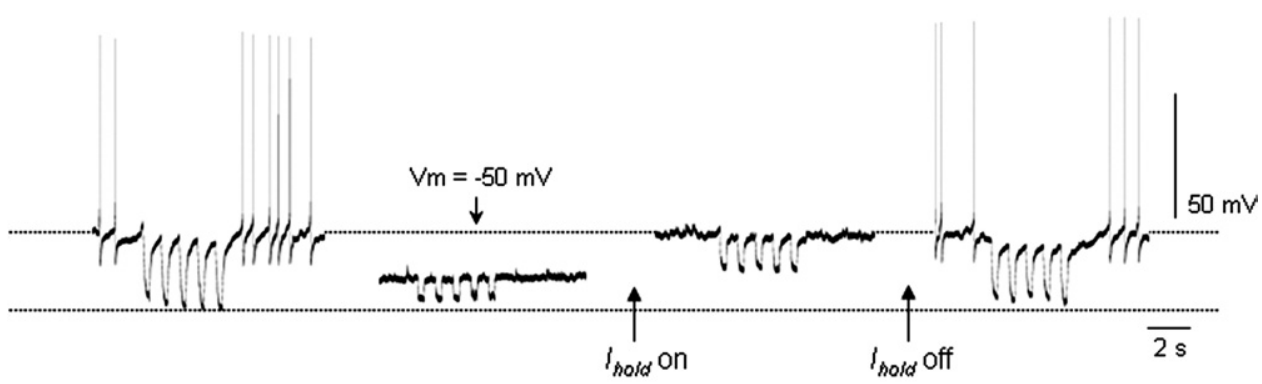

C

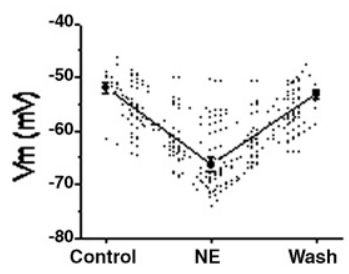

D

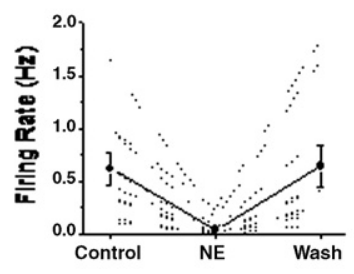

E

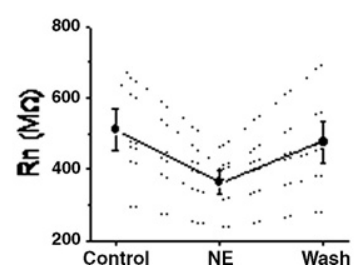

Fig. 5. Effect of NE on NAergic neurons. (A) Bath application of $10 \mu \mathrm{M} \mathrm{NE}$ (horizontal bar) causes hyperpolarization of the Vm and completely blocks spontaneous firing in a NAergic neuron. (B) A hyperpolarizing current pulse of $40 \mathrm{pA}$ was applied to estimate the Rn (trace i) in a NAergic neuron; bath application of NE caused hyperpolarization of the $\mathrm{Vm}$ and a reduction in the membrane voltage response to hyperpolarizing current pulses (trace ii). When depolarizing current $\left(\mathrm{I}_{\text {hold }}\right)$ was applied to hold the $\mathrm{Vm}$ at the resting level, a decrease in the $\mathrm{Rn}$ and neuronal excitability was seen, as the voltage response to a $40 \mathrm{pA}$ current pulse was reduced (indicating a decrease in $\mathrm{Rn}$ ) and there was no spontaneous firing (indicating a decrease in excitability) (trace iii). The effect of NE was completely recovered after a 10 min wash-out (trace iv). (C-E) Summarized results for the effect of NE on Vm hyperpolarization (C), spontaneous firing (D) and the $\mathrm{Rn}(\mathrm{E})$. Each dotted line represents the data for one neuron and the symbol and solid line are the mean \pm standard error.

lateral to the LC and the thickness of our slices was $300 \mu \mathrm{m}$, it is unlikely that the recorded neurons in our study were in the LC.

\section{Physiological and morphological properties of NAergic neurons in the $A 7$ area}

All the DBH-ir neurons, presumed to be NAergic neurons, recorded in this study had distinct physiological properties, including lacking a depolarizing voltage sag/rebound AP in response to injection of a hyperpolarizing current pulse, generating $\mathrm{Ca}^{2+}$-mediated APs with an amplitude overshooting $0 \mathrm{mV}$ (see online Supplemental Fig. 2), having a prominent A-type potassium current $\left(I_{A}\right)$ characterized by a voltage-dependent delay in the initiation of the first AP after injection of a depolarizing current pulse (Burdakov and Ashcroft, 2002; Burdakov et al., 2004) and being hyperpolarized by NE. They had a somata size of $20-25 \mu \mathrm{m}$. All of these features are compatible with those of neurons in the LC and subcoeruleus nuclei, which have all the physiological properties mentioned above and a medium to large somata size (long axis 25-30 $\mu \mathrm{m}$ ) (Williams et al., 1984; Brown et al., 2006).

\section{Effect of Sub-P on neurons in the A7 area}

Previous studies have demonstrated that NAergic neurons in the A7 area receive Sub-P-releasing nerve terminals from neurons in the RVM (Yeomans and Proudfit, 1990, 1992), ventrolateral PAG (Bajic et al., 2001) and LH (Holden et al., 2002). Antinociception can be directly induced by microinjection of Sub-P into the A7 catecholamine cell group (Yeomans and Proudfit, 1992) and this may result from activation of NAergic neurons by Sub-P, as direct synaptic contacts between Sub-P-releasing terminals and NAergic neurons have been identified at the electron microscopic level (Proudfit and Monsen, 1999; 


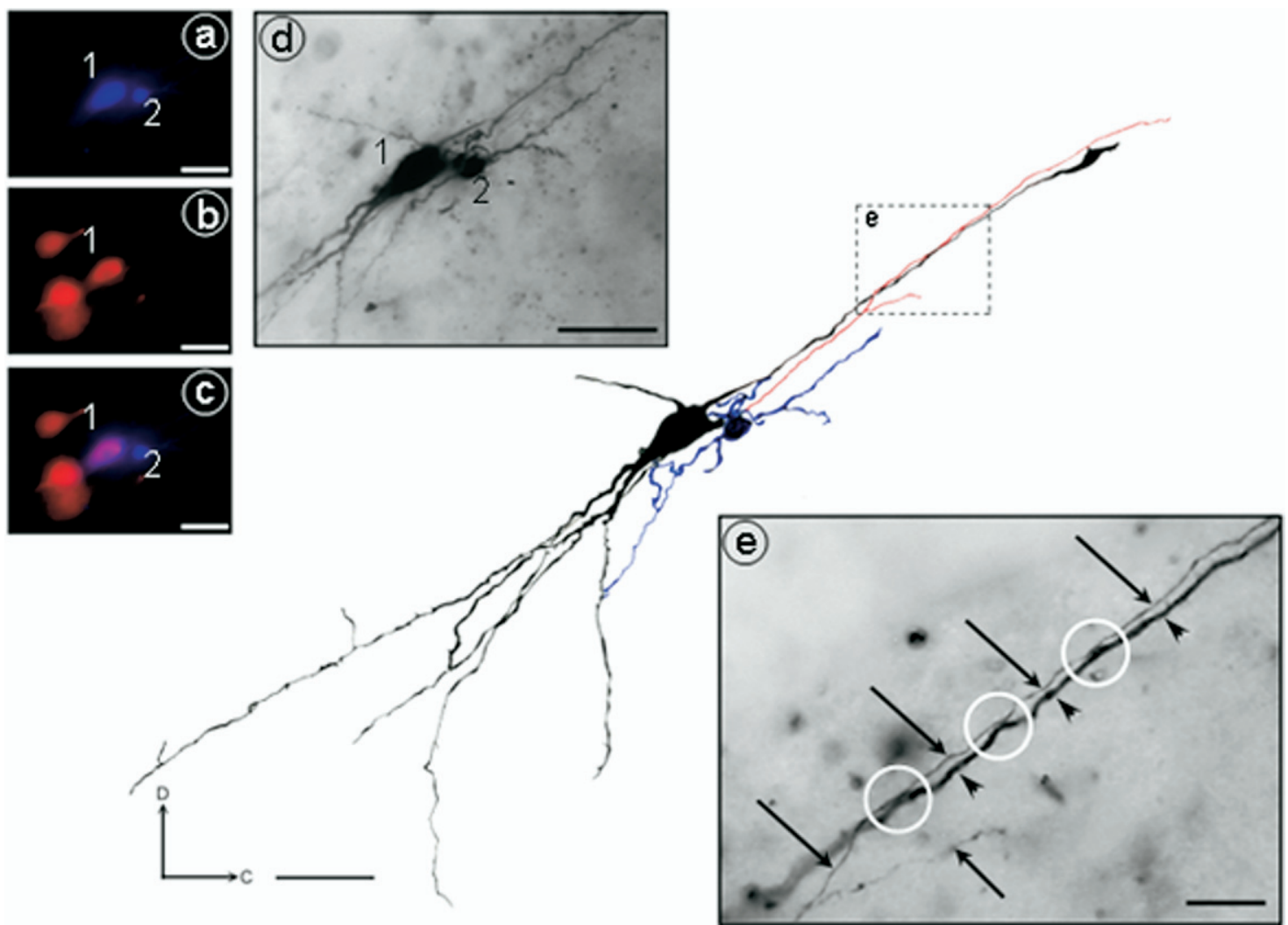

Fig. 6. Morphological evidence for possible synaptic connections between NAergic and non-NAergic neurons in the A7 area. Main diagram: Camera lucida drawing of a NAergic neuron (in black) and a non-NAergic neuron (dendrites and soma in blue and axon in red). Insets a-c show biocytin-AMCA-avidin-D labeling of the two recorded neurons (a), DBH immunostaining (b) and the merged image (c). Note the large cell (1) is DBH-ir, but the small cell (2) is not. Inset d shows subsequent staining with biocytin-HRP, which provides better resolution of the fine structure for camera lucida drawing. Inset e shows a high power micrograph of the dotted square and reveals clear physical contacts (white arrows) between the axon of the non-NAergic neuron (black arrows) and the dendrite of the NAergic neuron (arrowheads). Scale bars $=50 \mu \mathrm{m}$, except in inset e, where it is $10 \mu \mathrm{m}$.

Bajic et al., 2001). Consistent with this, there is immunohistochemical evidence for the expression of NK-1 receptors on NAergic neurons (Chen et al., 2000). In line with these observations, all NAergic neurons tested were excited by application of Sub-P and this effect was mimicked or blocked, respectively, by an NK-1 receptor agonist or antagonist, showing that Sub-P was acting predominantly at NK-1 receptors. Since the effect of Sub-P was tested under blockage of synaptic inputs, this suggests a postsynaptic effect, which is consistent with the observation that NK-1 receptors are expressed in TH-ir neurons in the A7 area (Chen et al., 2000). Although the effect was not significant, depolarization of the $\mathrm{Vm}$ by Sub-P was associated with a slight decrease in the Rn in NAergic neurons, suggesting that opening of cationic channels is involved in the effect of Sub-P (see also Oh et al., 2003). Since the NK-1 receptor is coupled to $\mathrm{Gq} / 11$ protein and since there is evidence that transient receptor potential (TRP) -like channels are activated by effectors following activation of G q/11 protein (Clapham, 2003; Oh et al., 2003; Moran et al., 2004), the excitatory effect of Sub-P on neurons in the A7 area might be due to the opening of TRP channels by NK-1 receptor activation.

In the present study, the effect of Sub-P was transient, as it started decaying before washout of Sub-P. This might be ascribed to the rapid desensitization of NK-1 receptors (Simmons, 2006) and/or to the downregulation of the targeted ion channels, both of which could be mediated by further downstream signaling effectors of the $G_{q / 11}$-IP3 signaling pathway. For example, it has been reported that canonical TRPC channels activated by group I metabotropic glutamate receptors ( $\mathrm{mGluRs}$ ) are downregulated by protein kinase $C$ activated by $G_{q / 11}-I_{3}$ signaling following the activation of group I mGluRs (Venkatachalam et al., 2003; Trebak et al., 2005). Another possible explanation for the transient effect of Sub-P could be the hyperpolarizing effect of NE on NAergic neurons, which is not only observed in the A7 area (this study), but also in the LC and other NAergic neurons (Williams et al., 1984; Lee et al., 1998; Grandoso et al., 2005; Brown et al., 2006). NE could be released from NAergic neurons excited by Sub-P, which could then counteract the excitatory effect of Sub-P on NAergic neurons. The hyperpolarization by NE was tested under blockage of synaptic inputs, again showing a postsynaptic effect. This hyperpolarizing effect of NE has been reported to act via $\alpha-2$ receptors in NAergic neurons in the LC or other subcoeruleus nuclei (Williams et al., 1984; Lee et al., 1998; Grandoso et al., 2005; Brown et al., 2006). 
A

Control

$0.2 \mu \mathrm{MSub} P$

Wash
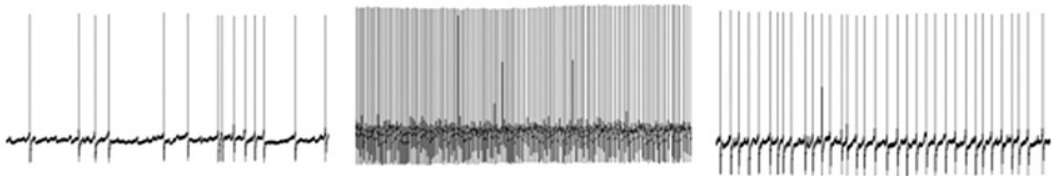

$\mathrm{J}_{10 \mathrm{~s}} 50 \mathrm{mV}$

A1 $0.2 \mu \mathrm{M}$ Sub-P

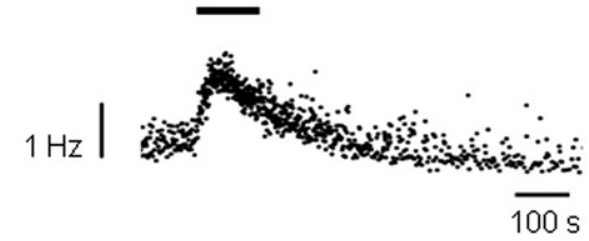

C

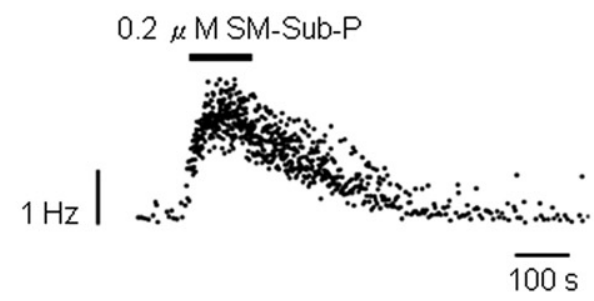

$0.2 \mu$ M SM-Sub-P

$10 \mu \mathrm{M}$ RP67580

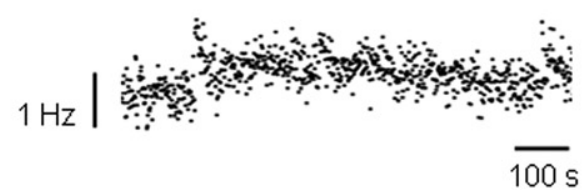

F

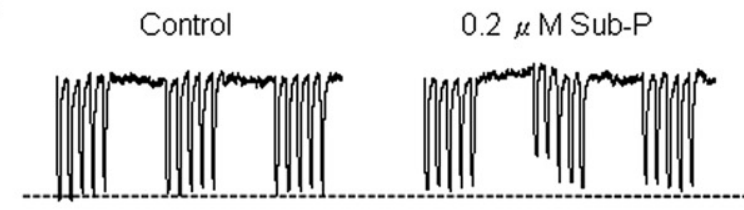

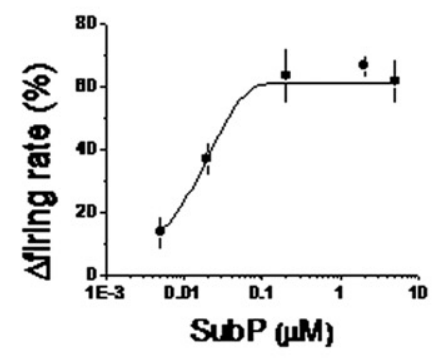

E

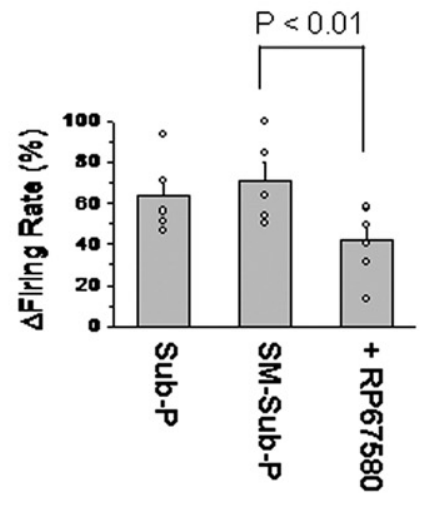

Wash

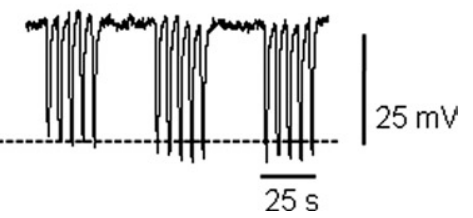

Fig. 7. Effect of Sub-P on NAergic neurons. (A) Examples of recordings made from a NAergic neuron in normal ACSF (left), $0.2 \mu M$ Sub-P (middle) and after 2 min of washout (right). Note the marked increase in firing rate caused by Sub-P when the entire time-course of recording is presented as instant frequency (A1). (B) The effect of Sub-P is dose-dependent. The results are the mean \pm S.E.M. for four cells at each concentration, except for $0.2 \mu \mathrm{M}$ (six cells). (C) The NK-1 receptor agonist, meSr-Sub-P, also causes a marked increase in firing rate of NAergic neurons. (D) The excitatory effect of Sub-P on a NAergic neuron is significantly attenuated by including $10 \mu \mathrm{M}$ RP67580, an NK-1 receptor blocker, in the ACSF. (E) Summarized results of the effect of Sub-P and meSr-Sub-P on the mean firing frequency of NAergic neurons and the attenuating effect of RP67580. Each circle represents a different NAergic neuron. $(F)$ With TTX and $\mathrm{Cd}^{2+}$ added to the ACSF to block the AP, Sub-P does not cause a significant change in the $\mathrm{Rn}$ of a NAergic neuron. A hyperpolarizing current pulse of $40 \mathrm{pA}$ was applied to estimate the $\mathrm{Rn}$ and a hyperpolarizing current was applied to hold the $\mathrm{Vm}$ at the resting level during application of Sub-P (middle trace).

\section{Non-NAergic (non DBH-ir) neurons in the A7 area}

In the LC, NAergic neurons are packed at a high density in the core of the nucleus, with some GABAergic interneurons surrounding the pericerulear dendritic zone (AstonJones et al., 2004). In the A7 area, however, the density of NAergic neurons was much lower. Furthermore, smallsized $(\sim 10-15 \mu \mathrm{m})$ non-NAergic interneurons were distrib- uted randomly between the NAergic neurons. Anatomical and pharmacological studies have suggested that these small non-NAergic interneurons might be GABAergic and that some might feed tonic inhibition to NAergic neurons (Holden et al., 1999; Nusier and Proudfit, 2000; Bajic et al., 2001). It is proposed that these GABAergic neurons are subject to inhibitory control by enkephalin released from 


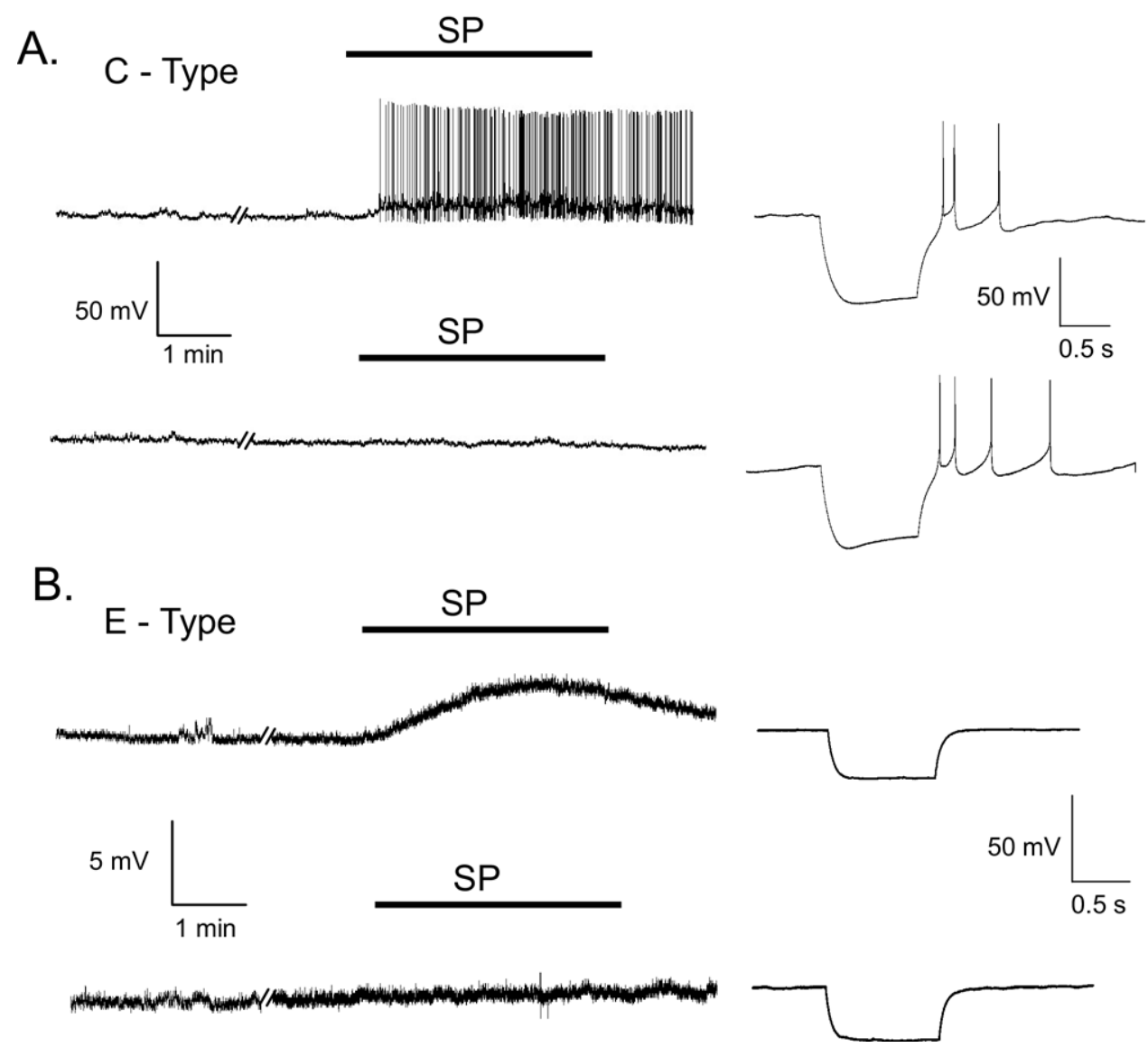

Fig. 8. Effect of Sub-P on type C and type E non-NAergic interneurons. (A, B) Recordings from two type C (A) and two type $E$ (B) interneurons. The trace on the right was recorded in normal ACSF and is shown at high speed. Note the voltage sag/rebound AP of the type $C$ neuron (right trace in A), which is not seen in the type $E$ neuron (right trace in B). None of the four neurons fired APs spontaneously. Upon application of Sub-P, one type C and one type $E$ neuron were depolarized (upper left traces in $A$ and $B$ ), while the other type $C$ and type $E$ neurons were not (lower left traces).

neurons in the RVM and ventrolateral PAG. Activation of the RVM and ventrolateral PAG would relieve the tonic inhibition of NAergic neurons in the A7 area, which, in turn, would increase NE release in the dorsal spinal cord and result in modulation of pain (Holden et al., 1999; Nusier and Proudfit, 2000; Bajic et al., 2001). Our morphological data are in support of this argument, as contacts between dendrites (or soma) of NAergic neurons and axonal varicosities of non-NAergic interneurons were identified in 14 of 22 paired NAergic and non-NAergic neurons recorded in the same slices.

In addition to GABA, glycine is a principal inhibitory neurotransmitter in the brainstem and spinal cord. Moreover, these two fast synaptic transmitters have been shown to be co-released from single inhibitory terminals in the spinal cord and brainstem (Yang et al., 1997; Jonas et al., 1998; Russier et al., 2002). In our preliminary study, we found that both spontaneous and evoked inhibitory synaptic currents were mediated by GABA and glycine in NAergic neurons in the $A 7$ area (Yang and Min, 2004). This raises the possibilities that non-NAergic neurons in the $A 7$ area could be GABAergic and/or glycinergic and that some could be excitatory, i.e. glutamatergic. Based on the firing patterns, the non-NAergic interneurons recorded in the $A 7$ area could be grouped into five types, suggesting the existence of functional diversity. Information about the neurotransmitter release profile of different functional types of interneuron is important in understanding local neuronal control in the A7 area and the mechanism underlying the antinociceptive effect of the $A 7$ catecholamine cell group at the level of the local neuronal circuit. Most studies that have explored the role of the A7 catecholamine cell group in modulating antinociception have examined the response of behaving animals to noxious stimulation upon stereotaxic lesion or chemical excitation (e.g. stereotaxic injection of Sub-P) of the A7 catecholamine cell group (Yeomans and Proudfit, 1992; Holden et al., 1999). Nevertheless, stereotaxic lesion or chemical excitation made to $A 7$ catecholamine cell group would not only cause lesion or excitation of NAergic neurons, but also of local nonNAergic interneurons. Since there are possible synaptic connections between local non-NAergic interneurons and NAergic neurons, it is important to verify whereas the change in response to noxious stimulation upon lesion or excitation of the $A 7$ catecholamine cell group is caused directly by the lesion/excitation of NAergic neurons or of non-NAergic interneurons that make synaptic connection with NAergic neurons. For example, the present data 
showed that, in every type of interneuron tested, some interneurons responded to application of Sub-P and, in all of these, Sub-P caused depolarization of the $\mathrm{Vm}$ and an increase in firing rate; it is therefore possible that the previously reported antinociceptive effect of stereotaxic injection of Sub-P into the A7 catecholamine cell group might have resulted from excitation of NAergic neurons or interneurons by Sub-P. For a better understanding of this issue, systematic investigations are required to define in more detail the neuronal subtypes of non-NAergic neurons, the properties of possible synaptic connections between these interneurons and NAergic neurons and the possible effect of Sub-P and other endogenous opioid peptides on these synaptic connections.

Acknowledgments-Grant sponsor: National Science Council, Taiwan. Grant numbers NSC 95-2320-B-040-011-MY3 (H.-W.Y.), CSMC 88-OM-B-027 (H.-W.Y.) and NSC 94-2311-B-002-023 (M.-M.Y.). Some of the immunostaining study was performed by Miss Pei-Chien Hsu.

\section{REFERENCES}

Aimone LD, Jones SL, Gebhart GF (1987) Stimulation-produced descending inhibition from the periaqueductal gay and nucleus raphe magnus in the rat: Mediation by spinal monoamines but not opioids. Pain 31:123-136.

Aston-Jones G, Cohen JD (2005) An integrative theory of locus coeruleus- norepinephrine function: adaptive gain and optimal performance. Annu Rev Neurosci 28:403-450.

Aston-Jones G, Zhu Y, Card JP (2004) Numerous GABAergic afferents to the locus coeruleus in the pericerulear dendritic zone: possible interneuronal pool. J Neurosci 24:2313-2321.

Bajic D, Van Bockstaele EJ, Proudfit HK (2001) Ultrastructure analysis of ventro-lateral periaqueductal gray projections to the A7 catecholamine cell group. Neuroscience 104:181-197.

Brown RE, Winston S, Basheer R, Thakkar MM, Mccarley RW (2006) Electrophysiological characterization of neurons in the dorsolateral pontine rapid-eye-movement induction zone of the rat: intrinsic membrane properties and response to carbachol and orexins. Neuroscience 143:739-755.

Burdakov D, Ashcroft FM (2002) Cholecystokinin tunes firing of an electrically distinct subset of accurate nucleus neurons by activating A-type potassium current. J Neurosci 22:6380-6387.

Burdakov D, Alexopoulos H, Vincent A, Ashcroft FM (2004) Lowvoltage-activated A-current controls the firing dynamics of mouse hypothalamic orexin neurons. Eur J Neurosci 20:3281-3285.

Burnett A, Gebhart GF (1991) Characterization of descending modulation of nociception from the A5 cell group. Brain Res 546: 271-281.

Chen L-W, Wei LC, Liu H-L, Rao Z-R (2000) Noradrenergic neurons expressing substance $P$ receptor (NK1) in the locus coeruleus complex: a double immunofluorescent study in the rat. Brain Res 873:155-159.

Clark FM, Proudfit HK (1991a) The projection of noradrenergic neurons in A7 catecholamine cell group to the spinal cord in rat demonstrated by antrograde tracing combined immunohistochemistry. Brain Res 547:279-288.

Clark FM, Proudfit HK (1991b) Projection of neurons in the ventromedial medulla to pontine catecholamine cell groups involved in the modulation of nociception. Brain Res 540:105-111.

Clapham DE (2003) TRP channels as cellular sensors. Nature 426:517-524.

Dahlström A, Fuxe K (1964) Evidence for the existence of monoaminecontaining neurons in the central nervous system. I. Demonstration of monoamines in the cell bodies of brainstem neurons. Acta Physiol Scand 232(Suppl 62):1-55.

Danzebrink RM, Gebhart GF (1990) Antinociceptive effects of intrathecal adrenoceptor agonists in a rat model of visceral nociception. J Pharmacol Exp Ther 253:698-705.

Dennis SG, Melzack R, Gutman S, Boucher F (1980) Pain modulation by adrenergic agents and morphine as measured by three pain tests. Life Sci 26:1247-1259.

Grandoso L, Torrecilla M, Pineda J, Ugedo L (2005) alpha(2)-Adrenoceptor involvement in the in vitro inhibitory effect of citalopram on a subpopulation of rat locus coeruleus neurons. Eur J Pharmacol 517(1-2):51-58.

Holden JE, Proudfit HK (1998) Enkephalin neurons that project to the A7 catecholamine cell group are located in brainstem nuclei that modulate nociception: ventromedial medulla. Neuroscience 83: 929-947.

Holden JE, Schwartz EJ, Proudfit HK (1999) Microinjection of morphine in the A7 catecholamine cell group produces opposing effects on nociception that are mediated by alpha1- and alpha2adrenoceptors. Neuroscience 83:979-990.

Holden JE, Van Poppel AY, Thomas S (2002) Antinociception from lateral hypothalamic stimulation may be mediated by NK1 receptors in the A7 catecholamine cell group in rat. Brain Res 953: 195-204.

Iwamoto ET, Marion L (1993) Adrenergic, serotonergic and cholinergic components of nicotinic antinociception in rats. J Pharmacol Exp Ther 265:777-789.

Jensen TS, Yaksh TL (1986) Examination of spinal monoamine receptors through which brainstem opiate-sensitive systems act in the rat. Brain Res 363:315-330.

Jonas P, Bischofberger J, Sandkühler J (1998) Corelease of two fast neurotransmitters at a central synapse. Science 281:419-424.

Jones SL (1991) Descending noradrenergic influences on pain. Prog Brain Res 88:381-394.

Kuraishi Y, Hirota N, Satoh M, Takagi H (1985) Antinociceptive effects of intrathecal opioids, noradrenaline and serotonin in rats: mechanical and thermal algesic tests. Brain Res 326:168-171.

Kwiat GC, Basbaum Al (1990) Organization of tyrosine hydroxylaseimmunoreactive and serotonin-immunoreactive brainstem neurons with axon collaterals to the periaqueductal gray and the spinal cord in the rat. Brain Res 528:83-94.

Kwiat GC, Basbaum Al (1992) The origin of brainstem noradrenergic and serotonergic projections to the spinal cord dorsal horn in the rat. Somatosens Mot Res 9:157-173.

Lee A, Rosin DL, Van Bockstaele EJ (1998) Ultrastructural evidence for prominent postsynaptic localization of alpha2C-adrenergic receptors in catecholaminergic dendrites in the rat nucleus locus coeruleus. J Comp Neurol 394:218-229.

Min M-Y, Hsu P-C, Yang H-W (2004) Whole cell recording from neurons located in pontine a7 nucleus in rat brainstem slices. Program no. 521.10. Soc Neurosci, http://www.snf.org.

Min M-Y, Hsu P-C, Yang H-W (2003) The physiological and morphological characteristics of interneurons caudal to the trigeminal motor nucleus in rats. Eur J Neurosci 18:2981-2998.

Min M-Y, Hsu P-C, Lu H-W, Lin C-J, Yang H-W (2007) Postnatal development of noradrenergic terminals in the rat trigeminal motor nucleus: a light and electron microscopic immunocytochemical analysis. Anat Rec 290:96-107.

Moran MM, Xu H, Clapham DE (2004) TRP ion channels in the nervous system. Curr Opin Neurobiol 14:362-369.

Nag S, Mokha SS (2004) Estrogen attenuates antinociception produced by stimulation of Kolliker-Fuse nucleus in the rat. Eur J Neurosci 20:3203-3207.

Nusier K, Proudfit HK (2000) Bidirectional modulation of nociception by GABA neurons in the dorsolateral pontine tegmentum that tonically inhibit spinally projecting noradrenergic $A 7$ neurons. Neuroscience 96:773-783. 
Oh EJ, Gover TD, Cordoba-Rodriguez R, Weinreich D (2003) Substance $\mathrm{P}$ evokes cation currents through TRP channels in HEK393 cells. J Neurophysiol 90:2069-2073.

Paxinos G, Watson C (1998) The rat brain in stereotaxic coordinates. San Diego: Academic Press.

Peng YB, Lin Q, Willis D (1996) Involvement of alpha-2 adrenoceptors in the periaqueductal gray-induced inhibition of dorsal horn cell activity in rats. J Pharmacol Exp Ther 278:125-135.

Pertovaara A (2006) Noradrenergic pain modulation. Prog Neurobiol 80:53-83.

Proudfit HK, Clark FM (1991) The projection of locus coeruleus neurons to the spinal cord. Prog Brain Res 88:123-141.

Proudfit HK, Monsen M (1999) Ultrastructural evidence that substance $P$ neurons form synapses with noradrenergic neurons in the A7 catecholamine cell group that modulate nociception. Neuroscience 91:1499-1531.

Reddy SV, Yaksh TL (1980) Spinal noradrenergic terminal system mediates antinociception. Brain Res 189:391-401.

Russier M, Kopysova IL, Ankri N, Ferrand N, Debanne D (2002) GABA and glycine co-release optimizes functional inhibition in rat brainstem motoneruons in vitro. J Physiol 541:123-137.

Simmons MA (2006) Functional selectivity of NK1 receptor signaling: peptide agonists can preferentially produce receptor activation or desensitization. J Pharmacol Exp Ther 319:907-913.

Stuart GJ, Dodt HU, Sakmann B (1993) Patch clamp recording from soma and dendrites of neurons in brain slices using infrared video microscopy. Pflügers Arch 423:511-518.

Takagi H, Shiomi H, Kuraishi Y, Fukui K, Ueda H (1979) Pain and the bulbospinal noradrenergic system: pain-induced increase in normetanephrine content in the spinal cord and its modification by morphine. Eur J Pharmacol 54:99-107.

Takano Y, Yaksh TL (1992) Characterization of the pharmacology of intrathecally administered a2 agonists and antagonists in rats. J Pharmacol Exp Ther 261:764-772.

Trebak M, Hempel N, Wedel BJ, Smyth JT, Bird GS, Putney JW Jr (2005) Negative regulation of TRPC3 channels by protein kinase C-mediated phosphorylation of serine 712. Mol Pharmacol 67: $558-563$.
Tyce GM, Yaksh TL (1981) Monoamine release from cat spinal cord by somatic stimuli: an intrinsic modulatory system. J Physiol (Lond) 314:513-529.

Venkatachalam K, Zheng F, Gill DL (2003) Regulation of canonical transient receptor potential (TRPC) channel function by diacylglycerol and protein kinase C. J Biol Chem 278:29031-29040.

Westlund KN, Coulter JD (1980) Descending projections of the locus coeruleus and subcoeruleus/medial parabrachial nuclei in monkey: axonal transport studies and dopamine-b-hydroxylase immunocytochemistry. Brain Res Rev 2:235-264.

Williams JT, North RA, Shefer SA, Nishi S, Egan TM (1984) Membrane properties of rat local coeruleus neurons. Neuroscience 13:137165.

Yang HW, Min MY (2004) The excitatory and inhibitory synaptic activity recorded from adrenergic neurons of A7 area in rats. Program No. 521.9. Soc Neurosci, http://www.snf.org.

Yang HW, Min MY, Appenteng K, Batten TFC (1997) Glycine-immunoreactive terminals in rat trigeminal motor nucleus: light- and electron-microscopic analysis of their relationship with motoneurons and GABA-immunoreactive terminals. Brain Res 749:301319.

Yeomans DC, Clark FM, Paice JA, Proudfit HK (1992) Antinociception induced by electrical stimulation of spinally projecting noradrenergic neurons in the A7 catecholamine cell group of the rat. Pain 48:449-461.

Yeomans DC, Proudfit HK (1990) Projection of substance P-immunoreactive neurons located in the ventromedial medulla to the $A 7$ noradrenergic nucleus of the rat demonstrated using retrograde tracing combined with immunohistochemistry. Brain Res 532: 329-332.

Yeomans DC, Proudfit HK (1992) Antinociception induced by microinjection of substance $P$ into the $A 7$ catecholamine cell group in the rat. Neuroscience 49:681-691.

\section{APPENDIX}

\section{Supplementary data}

Supplementary data associated with this article can be found, in the online version, at doi: 10.1016/j.neuroscience.2008.03.011. 